\title{
Keratitis-Ichthyosis-Deafness Syndrome, Atypical Connexin GJB2 Gene Mutation, and Peripheral T-Cell Lymphoma: More Than a Random Association?
}

\author{
Claudio Fozza, ${ }^{1}$ Fausto Poddie, ${ }^{2}$ Salvatore Contini, ${ }^{1}$ Antonio Galleu, ${ }^{1}$ \\ Francesca Cottoni, ${ }^{3}$ Maurizio Longinotti, ${ }^{1}$ and Francesco Cucca ${ }^{2}$ \\ ${ }^{1}$ Institute of Hematology, University of Sassari, 07100 Sassari, Italy \\ ${ }^{2}$ Institute of Medical Genetics, University of Sassari, 07100 Sassari, Italy \\ ${ }^{3}$ Institute of Dermatology, University of Sassari, 07100 Sassari, Italy \\ Correspondence should be addressed to Claudio Fozza, cfozza@uniss.it
}

Received 24 May 2011; Accepted 26 June 2011

Academic Editors: D. J. Allsup and T. Sonoki

Copyright ( $) 2011$ Claudio Fozza et al. This is an open access article distributed under the Creative Commons Attribution License, which permits unrestricted use, distribution, and reproduction in any medium, provided the original work is properly cited.

Keratitis-ichthyosis-deafness (KID) syndrome is a rare congenital disorder characterized by skin lesions, neurosensorial hypoacusia, and keratitis, usually due to the $\mathrm{c} \cdot 148 \mathrm{G} \rightarrow$ A mutation involving the connexin 26 gene. We report on a KID patient who showed the atypical c.101T $\rightarrow$ C mutation and developed a T-cell lymphoma so far never described in this group of patients.

\section{Introduction}

Keratitis-ichthyosis-deafness (KID) syndrome is a rare congenital disorder characterized by a variety of skin lesionsthat is, palmoplantar keratoderma, thickening of the skin, and erythematous verrucous lesions-neurosensorial hypoacusia, and keratitis with a variable degree of visual impairment [1]. Both sporadic and familial forms of the syndrome have been described, the latter usually showing a dominant pattern of inheritance [2]. The molecular lesion responsible for the syndrome typically involves the connexin 26 (Cx26) gene (GJB2). Most patients display the heterozygous $c .148 \mathrm{G} \rightarrow \mathrm{A}$ mutation causing the substitution of an aspartic acid for an asparagine at position 50 (p.Asp50Asn), while a few of them show the $\mathrm{c} .50 \mathrm{C} \rightarrow \mathrm{T}$ mutation, implying the substitution of a serine for a phenylalanine at position 17 (p.Ser17Phe) [2]. However, even a mutation in the connexin 30 (Cx30) gene (GJB6) has been found in a typical KID patient [3], thus suggesting a genetic heterogeneity of the syndrome. As connexins are a large family of small integral membrane proteins which influence tissue cornification by modulating the establishment of direct cell-cell communication through gap junction channels [4], it is likely that defects involving this class of proteins are at the basis of the wellknown increased incidence of squamous cell carcinoma in KID patients [5].

\section{Case Presentation}

Here we report on an adult patient with a typical KID syndrome who developed a peripheral T-cell lymphoma. It is worth noting that sequencing of GJB2 and GJB6 genes revealed only a Cx26 (GJB2) c.101T $\rightarrow$ C mutation, a variant usually associated with isolated hearing impairment $[6,7]$.

Briefly, the patient presented skin ichthyosis since his adolescence and in subsequent years developed severe bilateral hypoacusia and keratitis. The coexistence of such progressively worsening features pointed to the clinical diagnosis of KID syndrome. At that time, no molecular investigations were performed. The patient came to our attention in November 2007, when he was 65 years old, with diffuse lymphoadenopathy and splenomegaly $(122 \mathrm{~mm})$ associated to thrombocytopenia $\left(84 \times 10^{9} / \mathrm{L}\right)$, neutropenia $\left(1.4 \times 10^{9} / \mathrm{L}\right)$, and elevated lactate dehydrogenase level $(1578 \mathrm{U} / \mathrm{L})$ along with a worsening of his erythematosus desquamating cutaneous rash. After an inguinal node 


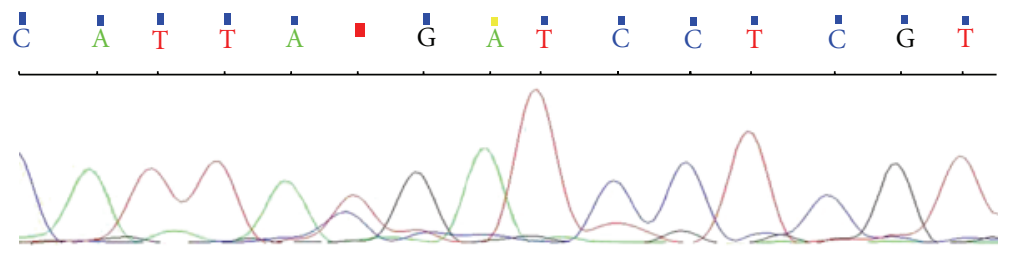

FIGURE 1: A search for mutations within the connexin 26 gene GJB2 showed the heterozygous c.101T $\rightarrow$ C mutation (in red in the figure) causing the substitution of a methionine residue for threonine at position 34 (p.Met34Thr).

TABLE 1: GJB2 forward and reverse primers.

\begin{tabular}{ll}
\hline GJB2 FW F1 & CATTCGTCTTTTCCAGAGCA \\
\hline GJB2 RV F1 & CACGTGCATGGCCACTAG \\
\hline GJB2 FW F2 & CGTGTGCTACGATCACTAC \\
\hline GJB2 RV F2 & AGCCTTCGATGCGGACCTT \\
\hline GJB2 FW F3 & ACCGGAGACATGAGAAGAAG \\
\hline GJB2 RV F3 & TTCCAGACACTGCAATCATG \\
\hline GJB2 FW F4 & TATGTCATGTACGACGGCT \\
\hline GJB2 RV F4 & TCTAACAACTGGGCAATGC \\
\hline
\end{tabular}

biopsy, a diagnosis of $\mathrm{CD} 3+$, $\mathrm{CD} 45 \mathrm{RO}+$, bcl2+, and $\mathrm{CD} 7+$ peripheral T-cell non-Hodgkin lymphoma (NHL) was made. Because of bone marrow involvement in trephine biopsy, the lymphoma stage resulted to be IV A with a high risk on the International Prognostic Index (IPI). Besides an infiltration by T-lymphoma cells, the skin biopsy showed epidermal cysts, hyperkeratotic lesions, and inflammatory nodules. The ophttalmoscopic and audiometric evaluations showed bilateral neurosensorial hypoacusia and superficial punctate keratitis. All these findings being compatible with a fully expressed KID phenotype, the GJB2 gene sequencing was firstly performed. Briefly, after genomic DNA extraction from peripheral blood following the standard salting-out procedures, GJB2 was amplified by PCR using the primers reported in Table 1. PCR products were then sequenced on an ABI Prism 3130 genetic analyzer by using BigDye Terminator v3.1 (Applied Biosystems) showing the heterozygous c.101T $\rightarrow \mathrm{C}$ mutation (Figure 1), which causes the substitution of a methionine residue for threonine at position 34 (p.Met34Thr, briefly M34T). Both the GJB2 c. $148 \mathrm{G} \rightarrow \mathrm{A}$ and $\mathrm{c.50C} \rightarrow \mathrm{T}$ gene mutations usually found to be associated with KID syndrome [2] were excluded. The sequencing analysis was then extended to the Cx30 GJB6 coding gene but failed to reveal any further mutation.

Our patient was treated with a combination of chemotherapy including Cyclophosphamide, Doxorubicin, Vincristine, and Prednisone and immunotherapy with Alemtuzumab. After a partial response, the patient died of Cytomegalovirus pneumonia 7 months after the diagnosis of T-cell lymphoma.

\section{Discussion}

The present case deserves some comments. Firstly, the M34T mutation causing the substitution of a methionine residue for threonine at position 34 (p.Met34Thr) has never been described in patients with typical KID syndrome, whereas it has already been found in a homozygous as well as in a double heterozygous state in subjects with isolated hearing impairment. However, even in these cohorts this mutation was reported with extremely low frequencies $[6,7]$. In addition, as the M34T variant has an allele frequency of about $1 \%$ even the in the whole European healthy population [8], we ought to conclude that the pathogenetic role of the M34T variant in our KID patient has still to be proved. Secondly, an increased susceptibility to cutaneous cancer has been reported in subjects with KID syndrome [5]. Considering that the CX26 gene modulates the cadherin expression [9], it is probable that such a susceptibility may be related to the cadherin downregulation described in approximately $70 \%$ of squamous cell carcinoma patients [10]. On the other hand T-cell NHLs are rare malignancies accounting for $10 \%$ to $15 \%$ of all NHLs [11]. Cadherin is expressed and functionally active even in T-lymphoma cells, implying a possible involvement in the mechanisms of lymphoma cell dissemination to skin and central nervous system [12]. Therefore, the coexistence of KID syndrome and $\mathrm{T}$-cell lymphoma may be more than a coincidence. In the same way as the gene sequencing of GJB2 and GJB6, with the exception of the M34T variant, did not reveal any of the molecular defects typical of KID syndrome, we are tempted to conclude that such an association of three extremely rare conditions in the same patient might not be merely accidental.

\section{References}

[1] B. A. Skinner, M. C. Greist, and A. L. Norins, "The keratitis, ichthyosis, and deafness (KID) syndrome," Archives of Dermatology, vol. 117, no. 5, pp. 285-289, 1981.

[2] J. Mazereeuw-Hautier, E. Bitoun, J. Chevrant-Breton et al., "Keratitis-ichthyosis-deafness syndrome: disease expression and spectrum of connexin 26 (GJB2) mutations in 14 patients," British Journal of Dermatology, vol. 156, no. 5, pp. 1015-1019, 2007.

[3] A. Y. Jan, S. Amin, P. Ratajczak et al., "Genetic heterogeneity of KID syndrome: identification of a Cx30 gene (GJB6) mutation in a patient with KID syndrome and congenital atrichia," Journal of Investigative Dermatology, vol. 122, no. 5, pp. 11081113, 2004.

[4] G. Richard, "Connexins: a connection with the skin," Experimental Dermatology, vol. 9, no. 2, pp. 77-96, 2000.

[5] J. J. Grob, A. Breton, J. L. Bonafe et al., "Keratitis, ichthyosis and deafness (KID) syndrome. Vertical transmission and 
death from multiple squamous cell carcinomas," Archives of Dermatology, vol. 123, no. 6, pp. 777-782, 1987.

[6] H. Azaiez, G. P. Chamberlin, S. M. Fischer et al., "GJB2: the spectrum of deafness-causing allele variants and their phenotype," Human Mutation, vol. 24, no. 4, pp. 305-311, 2004.

[7] A. C. Batissoco, R. S. Abreu-Silva, M. C. Braga et al., "Prevalence of GJB2 (connexin-26) and GJB6 (connexin-30) mutations in a cohort of 300 Brazilian hearing-impaired individuals: implications for diagnosis and genetic counseling," Ear and Hearing, vol. 30, no. 1, pp. 1-7, 2009.

[8] D. Feldmann, F. Denoyelle, N. Loundon et al., "Clinical evidence of the nonpathogenic nature of the M34T variant in the connexin 26 gene," European Journal of Human Genetics, vol. 12, no. 4, pp. 279-284, 2004.

[9] A. B. Stoler, F. Stenback, and A. Balmain, "The conversion of mouse skin squamous cell carcinomas to spindle cell carcinomas is a recessive event," Journal of Cell Biology, vol. 122, no. 5, pp. 1103-1117, 1993.

[10] S. Koseki, T. Aoki, S. Ansai et al., "An immunohistochemical study of E-cadherin expression in human squamous cell carcinoma of the skin: relationship between decreased expression of E- cadherin in the primary lesion and regional lymph node metastasis," Journal of Dermatology, vol. 26, no. 7, pp. 416-422, 1999.

[11] J. M. Vose, "Peripheral T-cell non-Hodgkin's lymphoma," Hematology/Oncology Clinics of North America, vol. 22, no. 5, pp. 997-1005, 2008.

[12] K. Kawamura-Kodama, J. Tsutsui, S. T. Suzuki et al., "Ncadherin expressed on malignant $\mathrm{T}$ cell lymphoma cells is functional and promotes heterotypic adhesion between the lymphoma cells and mesenchymal cells expressing $\mathrm{N}$ cadherin," Journal of Investigative Dermatology, vol. 112, no. 1, pp. 62-66, 1999. 


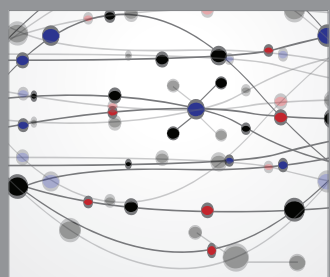

The Scientific World Journal
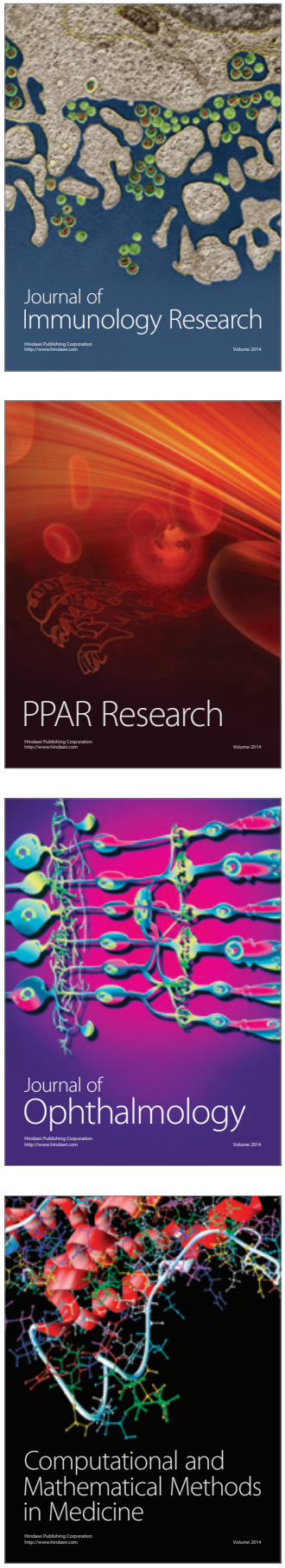

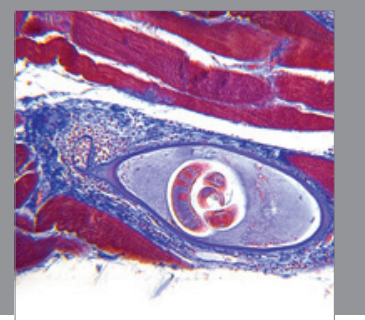

Gastroenterology

Research and Practice
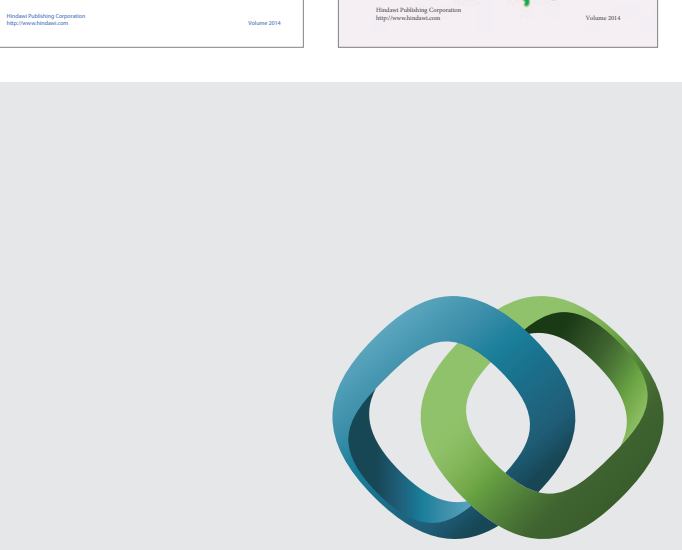

\section{Hindawi}

Submit your manuscripts at

http://www.hindawi.com
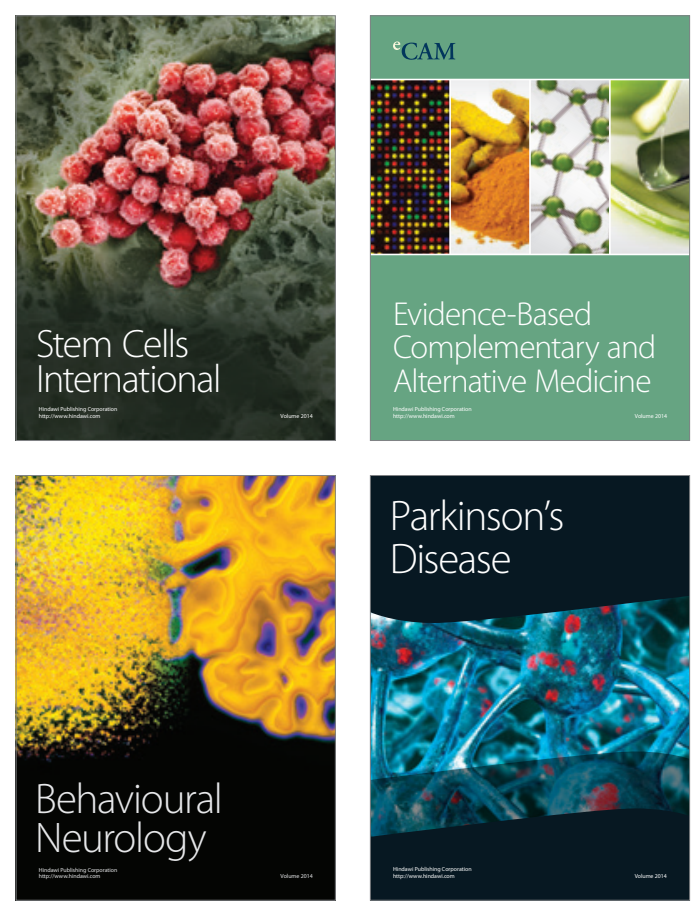

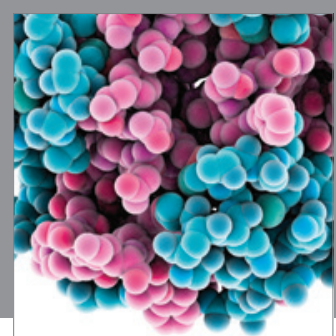

Journal of
Diabetes Research

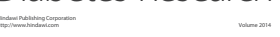

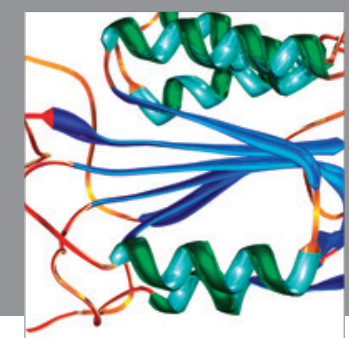

Disease Markers
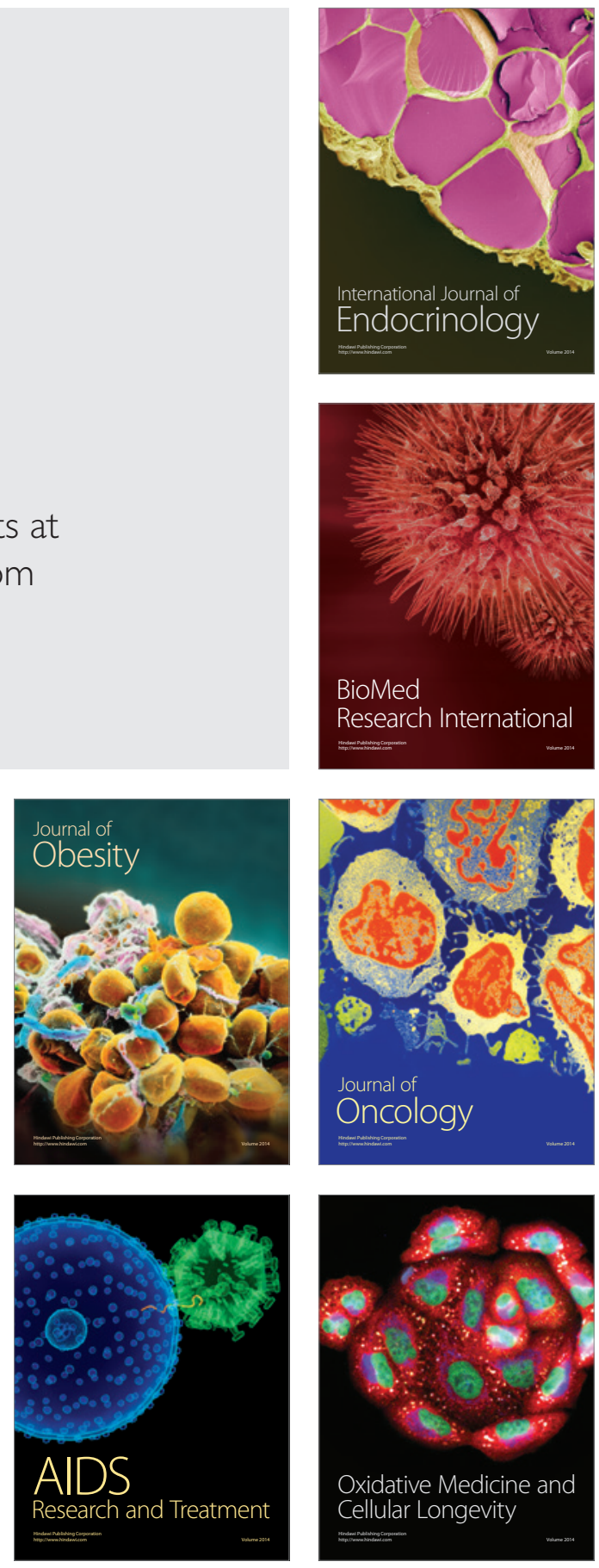